\title{
Vibro-acoustic response of FGM plates considering the thermal effects
}

\author{
Tieliang Yang ${ }^{1, \text { a }}$, Qibai Huang ${ }^{1, *}$
}

${ }^{1}$ State Key Laboratory of Digital Manufacturing Equipment and Technology, Huazhong University of Science and Technology, Wuhan 430074, PR China

aE-mail: yangtieliang@hust.edu.cn, ”Corresponding author: qbhuang@hust.edu.cn

Keywords: Vibro-acoustic response; FGM; Thermal environment

\begin{abstract}
In this paper, the vibro-acoustic response of functionally graded materials (FGM) plates subjected to thermal environment are investigated analytically. The governing equations of the FGM plate subjected to thermal environment are derived based on the classic plate theory through Hamilton's principle. The sound radiation of the FGM plate is calculated with Rayleigh integral. It is found that a dramatic discrepancy will occur if temperature dependent material properties are not taken into account. The temperature rise also has a significant effect on the vibro-acoustic response of the FGM plates.
\end{abstract}

\section{Introduction}

Functionally graded materials (FGM) are microscopically inhomogeneous composites in which material properties vary continuously and smoothly from one surface to another. Possessing a number of advantages such as eliminating interface problems and mitigating thermal stress concentrations leads to a wider application of FGMs in areas such as aircraft, space vehicles, nuclear plants [1]. Plate is one of the most widely used structural components in industrial applications. Sound radiation from plate structures is a practical engineering problem that has been studied extensively. However, only few works can be found in the literature focused on the sound radiation characteristics of FGM plates [2,3]. In addition, the FGM structures are always used in extreme thermal environment, and thermal environment may change the stiffness and dynamic response of the FGM structures. Therefore, it's significant to consider the thermal effects when dealing with the sound radiation of FGM plates in thermal environment.

The early literature focused on the structure dynamic characteristics under thermal environment can date back to 1950s [4], and in recent years, more and more attention has been paid to this problem. Jeyaraj et al. [5] presented a numerical simulation study on the vibration and acoustic response characteristics of a multilayered viscoelastic sandwich plate in a thermal environment. Geng et al. [6] investigated the dynamic and acoustic responses of a simply supported rectangular plate in thermal environments. $\mathrm{Li}$ and $\mathrm{Yu}$ [7] studied the vibration and acoustic responses of the sandwich panels in a high temperature environment based on the piecewise low order shear deformation theory. More recently, Du et al.[8] carried out an investigation on the dynamic characteristics of a laminated plate under temperature gradient, and it is found that the initial thermal deformation as well as the thermal stress have to be considered together in simulation of the dynamical response for thermal structure.

This paper presents an analytical investigation on the vibro-acoustic response of FGM plates in thermal environment. The governing equations of the FGM plate subjected to thermal environment are derived based on the classic plate theory through Hamilton's principle, and the acoustic response of the FGM plate is obtained with the use of the Rayleigh integral. Accuracy of the results is examined by comparing the obtained results of the present formulation with that available in the literature. Finally, some parametric studies are conducted to investigate the acoustic characteristics of FGM plates in thermal environment. 


\section{Theory and formulation}

\subsection{Effective properties of FGM plate}

Consider a rectangular FGM plate of length $a$, width $b$ and uniform thickness $h$. The FGM plate is made of a mixture of a metal and a ceramic, and the material properties of FGM plate are assumed to vary smoothly and continuously through the thickness from the ceramic surface to metal surface. The effective material properties can be defined by the Voigt model, according to which the effective material properties $P(\mathrm{z})$ such as Young's modulus $E$, density $\rho$, Poisson's ratio $v$, thermal conductivity $\lambda$, and thermal expansion $\alpha$ are expressed in terms of the material properties and volume fractions of constituents [9]

$$
P(z)=P_{\mathrm{c}} V_{\mathrm{c}}+P_{m} V_{m}
$$

where $P_{\mathrm{m}}$ and $P_{\mathrm{c}}$ denote the specific material properties of the metallic and ceramic constituents, respectively, and $V_{\mathrm{m}}$ and $V_{\mathrm{c}}$ represent the volume fractions of the metallic and ceramic constituents, respectively. By applying the power law distribution, the volume fractions of ceramic and metal are assumed as

$$
V_{c}=(z / h+0.5)^{N}, V_{c}+V_{m}=1 \quad(-0.5 h \leq z \leq 0.5 h)
$$

where $N$ is a non-negative real number and called the power law index.

The temperature dependent material properties are considered and the corresponding properties are given by [9]

$$
P(T)=P_{0}\left(P_{-1} T^{-1}+1+P_{1} T+P_{2} T^{2}+P_{3} T^{3}\right)
$$

where $T$ is the temperature in Kelvin $T=T_{0}+\Delta T$, with $T_{0}$ the initial uniform temperature $T_{0}=300$ $\mathrm{K}$ (where the plate is assumed to be stress free), and $\Delta T$ the temperature change, $P_{0}, P_{-1}, P_{1}, P_{2}$, and $P_{3}$ are the temperature coefficients which are unique to the constituent materials. In this paper, it is assumed that the Young's moduli E, Poisson's ratio $v$, thermal expansion coefficient $\alpha$ of the FGM plate are temperature dependent, whereas mass density $\rho$ and thermal conductivity $\lambda$ are independent of the temperature.

It is assumed that no heat generation source exists within the plate, and the temperature variation occurs in the thickness direction only and one-dimensional temperature field is considered to be constant in the $x y$ - plane. the temperature distribution along the thickness can be obtained by solving the following steady-state heat transfer equation through the thickness of the plate [9]

$$
-\frac{d}{\mathrm{dz}}\left[\lambda(z) \frac{\mathrm{d} T}{\mathrm{dz}}\right]=0
$$

This equation is solved by imposing the boundary condition of $T=T_{t}$ at $z=h / 2$ and $T=T_{b}$ at $z=-h / 2$, then the solution of Eq.(4) can be written as [9]

$$
T(z)=T_{t}-\frac{T_{t}-T_{b}}{\int_{-h / 2}^{+h / 2} d z / \lambda(z, T)} \int_{-h / 2}^{z} \frac{d z}{\lambda(z, T)}
$$

and the temperature change is defined as $\Delta T=T_{t}-T_{b}$.

\subsection{Governing equations}

According to the classic plate theory, the displacement field at any point of the plate can be written as [10]

$$
u(x, y, z, t)=u_{0}(x, y, t)-z w_{0, x}, v(x, y, z, t)=v_{0}(x, y, t)-z w_{0, y}, w(x, y, z, t)=w_{0}(x, y, t)
$$

where $(u, v, w)$ are the displacement components along the $(x-, y-, z-)$ coordinates, respectively, $\left(u_{0}, v_{0}, w_{0}\right)$ are the displacement components of the middle plane along the $\left(x^{-}, y_{-}, z_{-}\right)$coordinates, respectively. The nonzero linear strains associated with the displacements are

$$
\varepsilon_{x x}=u_{0, x}-z w_{0, x x}, \varepsilon_{y y}=v_{0, y}-z w_{0, y y}, \gamma_{x y}=v_{0, x}+u_{0, y}-2 z w_{0, x y}
$$

where $\varepsilon_{x x}, \varepsilon_{y y}$ and $\gamma_{x y}$ are the strain components. The linear constitutive relations are

$$
\sigma_{x}=c_{11} \varepsilon_{x}+c_{12} \varepsilon_{y}, \sigma_{y}=c_{21} \varepsilon_{x}+c_{22} \varepsilon_{y}, \sigma_{x y}=c_{66} \gamma_{x y}
$$


where $c_{\mathrm{ij}}$ are the elastic coefficient which are given by

$c_{11}=c_{22}=E /\left(1-v^{2}\right), c_{12}=c_{21}=v E /\left(1-v^{2}\right), c_{66}=0.5 E /(1+v)$ by

According to the Hamilton's principle, the dynamic equations of the FGM plate can be derived

$$
\int_{t 1}^{t 2} \delta\left(U+V_{1}+V_{2}-T\right) \mathrm{d} t=0
$$

where $\delta$ is the variation operator, $T$ is the kinetic energy of the system, $U$ is the potential energy of the system, $V_{1}$ is the potential energy done by the external load $q$, and $V_{2}$ is the potential energy induced by the thermal effect. The $\delta U, \delta T, \delta V_{1}$ and $\delta V_{2}$ can be expressed as

$$
\begin{aligned}
& \delta U=\int_{A}\left(N_{x x} \delta \varepsilon_{x}+N_{y y} \delta \varepsilon_{y}+N_{x y} \delta \gamma_{x y}\right) \mathrm{dA}, \delta T=\delta \int_{V} \rho(z)\left(u_{, t}{ }^{2}+v_{, t}{ }^{2}+w_{, t}{ }^{2}\right) d V \\
& \delta V_{1}=-\int_{A} q \delta w d A, \delta V_{2}=\int_{A}\left(N_{x x}^{T} \frac{\partial w_{0}}{\partial x} \frac{\partial \delta w_{0}}{\partial x}+N_{x y}^{T} \frac{\partial w_{0}}{\partial x} \frac{\partial \delta w_{0}}{\partial y}+N_{y y}^{T} \frac{\partial w_{0}}{\partial y} \frac{\partial \delta w_{0}}{\partial y}\right) d A
\end{aligned}
$$

Where

$$
\begin{aligned}
& \left(N_{x x}, N_{y y}, N_{x y}\right)=\int_{-\frac{h}{2}}^{\frac{h}{2}}\left(\sigma_{x x}, \sigma_{y y}, \tau_{x y}\right) d z, \quad\left(M_{x x}, M_{y y}, M_{x y}\right)=\int_{-\frac{h}{2}}^{\frac{h}{2}}\left(\sigma_{x x}, \sigma_{y y}, \tau_{x y}\right) z d z \\
& \left(N_{x x}^{T}, N_{y y}^{T}\right)=-\int_{-0.5 h}^{0.5 h}\left(\alpha(z, T) \Delta T \frac{E(z, T)}{1-v(z, T)}\right) d z, N_{x y}^{T}=0
\end{aligned}
$$

Substituting Eq.(10) into Eq. (9), and collecting the $\delta u_{0}, \delta v_{0}$, and $\delta w_{0}$, then the following equations can be obtained

$$
\begin{aligned}
& \delta u_{0}: N_{x x, x}+N_{x y, y}=I_{0} u_{0, t t}-I_{1} w_{0, t t x} \\
& \delta v_{0}: N_{y y, y}+N_{x y, x}=I_{0} v_{0, t t}-I_{1} w_{0, t t y} \\
& \delta w_{0}: M_{x x, x x}+M_{y y, y y}+M_{x y, x y}-N_{x x}^{T} w_{0, x x}-N_{y y}^{T} w_{0, y y}+q=I_{0} w_{0, t t}-I_{2}\left(w_{0, t x x}+w_{0, t t y y}\right)+I_{1}\left(u_{0, t t x}+v_{0, t y y}\right)
\end{aligned}
$$

where $\left(I_{0}, I_{1}, I_{2}\right)$ are the stress resultants defined by

$$
\left(I_{0}, I_{1}, I_{2}\right)=\int_{-0.5 h}^{-0.5 h} \rho(z)\left(1, z, z^{2}\right) d z
$$

In this paper, only the simply supported boundary conditions are considered, and the state variables satisfying the simply supported boundary conditions are assumed as the following form

$$
\left\{\begin{array}{l}
u \\
v \\
w
\end{array}\right\}=\sum_{m=1}^{\infty} \sum_{n=1}^{\infty}\left\{\begin{array}{l}
\bar{u} \cos (m \pi x / a) \sin (n \pi y / b) \\
\bar{v} \sin (m \pi x / a) \cos (n \pi y / b) \\
\bar{w} \sin (m \pi x / a) \sin (n \pi y / b)
\end{array}\right\} \exp (i \omega t)
$$

By substituting Eq.(14) into Eq.(12), the governing equations of FGM plates in thermal environment can be derived. If the external load $q$ is known, and then the displacements of the plate can be obtained with the use of the governing equations.

\subsection{Sound radiation power}

The radiated sound power can be obtained by integrating the acoustic intensity over the surface of the plate [11]

$$
\bar{W}=\int_{S} 0.5 \operatorname{Re}\left(\dot{w}^{*}\left(\mathbf{r}_{s}\right) p(\mathbf{r})\right) d S
$$

where Re and superscript * denote the real part and the complex conjugate, respectively, $p(\mathbf{r})$ is the complex pressure amplitude at location $\mathrm{r}, \dot{w}\left(\mathbf{r}_{s}\right)$ is the surface complex velocity. For a plate set in an infinite rigid baffle, the acoustic pressure $p(\mathbf{r})$ at any field point $\mathbf{r}$ can be expressed in terms of surface complex velocity according to Rayleigh integral [11].The radiated sound power is usually written in the form of sound power level in decibel, which is defined by

$$
S P L=10 \log \left(\bar{W} / \bar{W}_{0}\right)
$$

with $\bar{W}_{0}$ is the reference power and $\bar{W}_{0}=1 \times 10^{-12} \mathrm{~W}$. 


\section{Results and discussions}

We first validate the formulation in this paper. The example is sound radiation from a rectangular Aluminum plate, which is taken from Geng et al. [6]. The material properties are the same as that in Geng et al. [6]. Table 1 shows the first five natural frequencies of the plate subjected to temperature rise of $\Delta T=45^{\circ} \mathrm{C}$. The comparison of the results of the sound radiation power of the plate obtained by the present formulation and Geng et al. [6] is shown in Figure 1. As can be seen from Table 1 and Figure 1, the results obtained from the present formulation and the model of Geng et al. [6] are in good agreement, which validates the present formulation in this paper.

Tab. 1 The first five natural frequencies of the plate with thermal load of $\Delta T=45^{\circ} \mathrm{C}$.

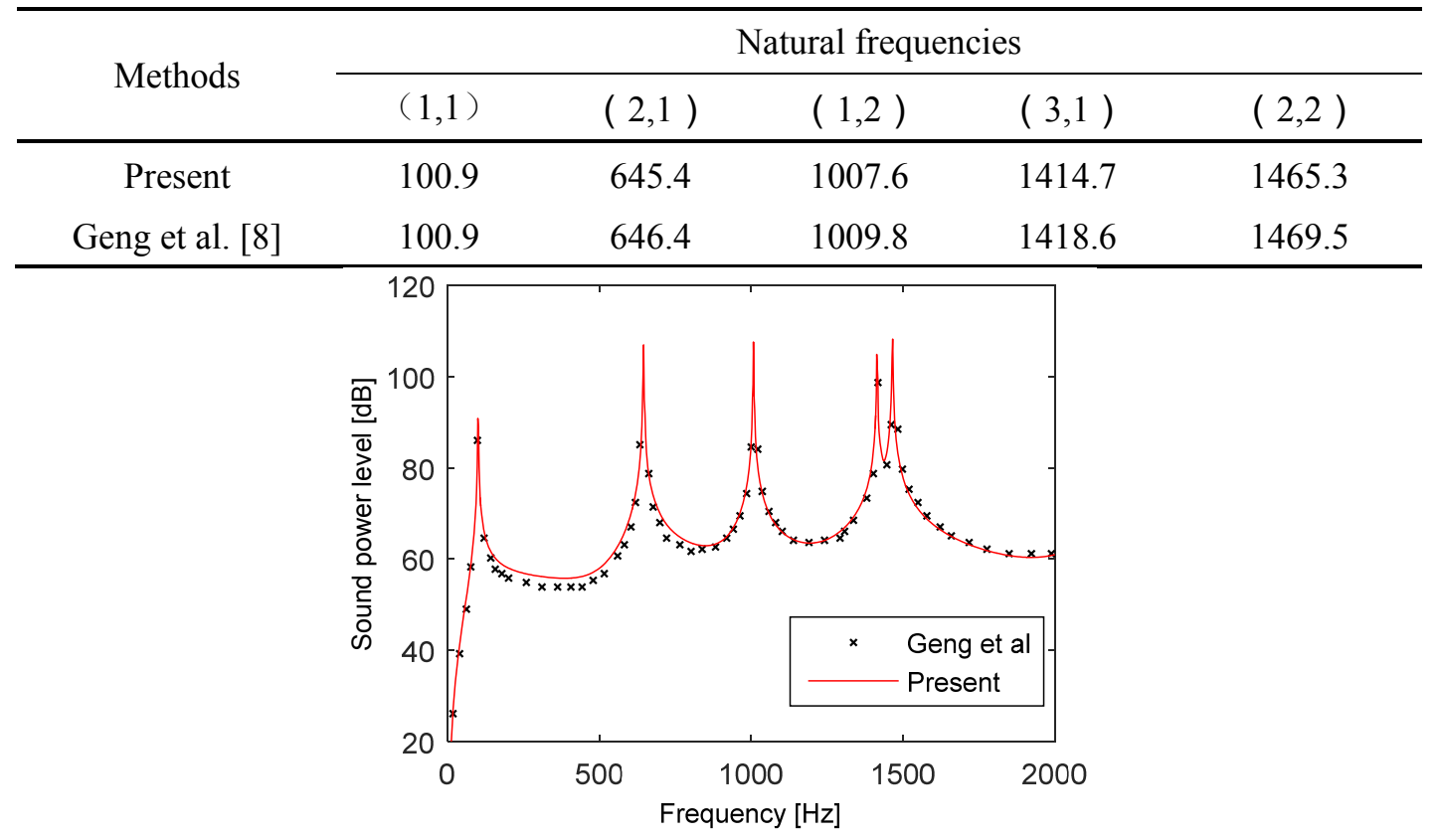

Fig. 1 Sound radiation power of the plate with thermal load of $\Delta T=45^{\circ} \mathrm{C}$.

Table 2 Temperature dependent material coefficients for ceramics and metals[9]

\begin{tabular}{ccccccc}
\hline Material & Properties & $P_{0}$ & $P_{-1}$ & $P_{1}$ & $P_{2}$ & $P_{3}$ \\
\hline SUS304 & $E(\mathrm{~Pa})$ & $201.04 \times 10^{9}$ & 0 & $3.079 \times 10^{-4}$ & $-6.534 \times 10^{-7}$ & 0 \\
& $v$ & 0.28 & 0 & 0 & 0 & 0 \\
& $\rho\left(\mathrm{kg} / \mathrm{m}^{3}\right)$ & 8166 & 0 & 0 & 0 & 0 \\
& $\alpha(1 / \mathrm{K})$ & $12.33 \times 10^{-6}$ & 0 & $8.086 \times 10^{-4}$ & 0 & 0 \\
$\mathrm{Si}_{3} \mathrm{~N}_{4}$ & $\lambda(\mathrm{W} / \mathrm{mK})$ & 12.04 & 0 & 0 & 0 & 0 \\
& $E(\mathrm{~Pa})$ & $348.43 \times 10^{9}$ & 0 & $-3.07 \times 10^{-4}$ & $2.160 \times 10^{-7}$ & $-8.946 \times 10^{-11}$ \\
& 0 & 0.28 & 0 & 0 & 0 & 0 \\
& $\rho\left(\mathrm{kg} / \mathrm{m}^{3}\right)$ & 2370 & 0 & 0 & 0 & 0 \\
& $\alpha(1 / \mathrm{K})$ & $5.872 \times 10^{-6}$ & 0 & $9.095 \times 10^{-4}$ & 0 & 0 \\
& $\lambda(\mathrm{W} / \mathrm{mK})$ & 9.19 & 0 & 0 & 0 & 0 \\
\hline
\end{tabular}

In the following, the developed formulation is thus deployed to carry out several parametric studies to examine the vibration and acoustic response of FGM plates in thermal environments. A rectangular $\mathrm{Si}_{3} \mathrm{~N}_{4} / \mathrm{SUS} 304 \mathrm{FGM}$ plate, simply supported on all edges with dimensions of $0.4 \mathrm{~m} \times$ $0.3 \mathrm{~m} \times 0.01 \mathrm{~m}$ is considered for the following detailed investigations. A point force of $1 \mathrm{~N}$ is applied on the corner of the plate $(x=0.1 \mathrm{~m}, y=0.1 \mathrm{~m})$ as the external load. The temperature dependent material properties of $\mathrm{Si}_{3} \mathrm{~N}_{4} / \mathrm{SUS} 304$ are given in Table 2. The plate is assumed to be vibrating in air. For the sake of convenience, the air density is taken to be $\rho_{0}=1.21 \mathrm{~kg} / \mathrm{m}^{3}$, and the 
speed of sound in the air is taken as $c_{0}=343 \mathrm{~m} / \mathrm{s}$. In addition, a damping loss factor of 0.01 is taken for the following calculations.

Fig. 2 illustrates the importance of considering the temperature dependent material properties in the calculation of sound radiation power of FGM plate in thermal environment. The thermal load $\Delta T=50 \mathrm{~K}$, and $\Delta T=130 \mathrm{~K}$ are considered. As shown in Figure 2, there is no considerable difference between the results of the sound radiation power with and without considering the temperature dependent material properties when the temperature rise is $\Delta T=50 \mathrm{~K}$, however, the discrepancy of that is distinct when the temperature rise approaches $\Delta T=130 \mathrm{~K}$. It can be seen from Figure 2 that the peaks of sound power level shift to lower frequency range when considering the temperature dependent material properties, which indicates that the natural frequencies of the FGM plates will be overestimated when the temperature dependent material properties are not taken into account. This is because that the temperature rise not only changes the pre-stress of the FGM plate, but also changes the material properties of the plate. When the temperature rise is small, the change of the material properties due to the temperature rise is not considerable; however, this factor cannot be neglected when the FGM plate subjected to an extreme temperature rise.

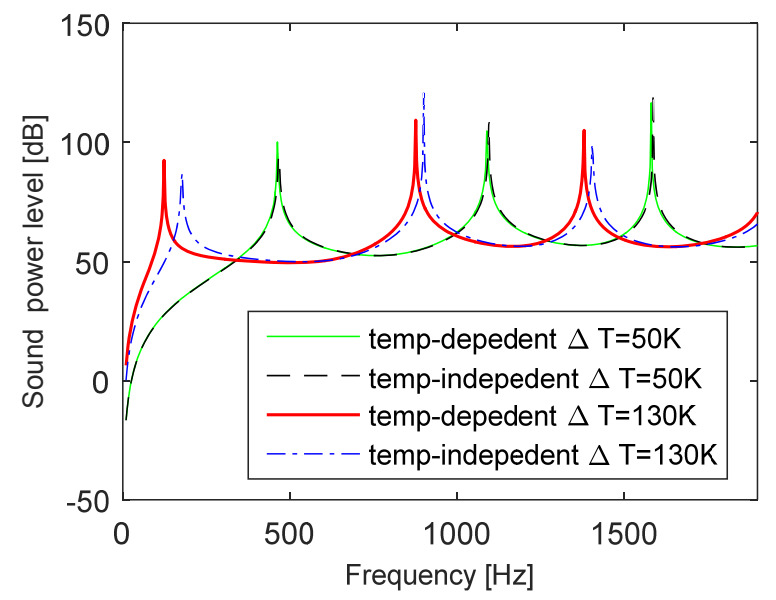

Fig. 2 Sound radiation power of the FGM plate: effect of considering the temperature dependent material properties.

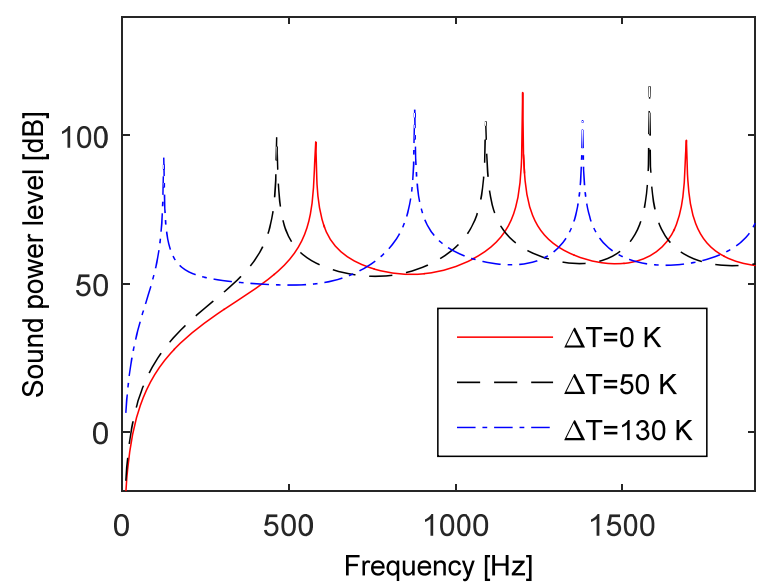

Fig. 3 Sound radiation power of the FGM plate: the effect of temperature rise.

Fig. 3 presents the effect of the temperature rise on sound radiation of FGM plates. The temperature change of $\Delta T=0 \mathrm{~K}, \Delta T=50 \mathrm{~K}$ and $\Delta T=130 \mathrm{~K}$ are considered. As shown in Fig. 3, the sound power level of FGM plate subjected to different temperature rise share a same tendency through the frequency band, however, it is observed that the corresponding peaks of the sound power level shift towards to lower frequency domain when the temperature rise increase. This is due to the fact that the corresponding natural frequencies decrease with the increase of temperature rise. The temperature rise not only softens the pre-stress of the FGM plate, but also decrease the Young's modulus, which result into a decrease of the natural frequency. 


\section{Conclusion}

The vibro-acoustic characteristics of functionally graded materials plates in thermal environment are presented in this paper. The classic plate theory is obtained to derive the governing equations of the FGM plate in thermal environment, and a good agreement has been achieved when compare the results of the present formulation with that available in the literature. The following conclusions can be made. The temperature dependent material properties have a significant importance on the vibro-acoustic response of the FGM plates in thermal environment. The natural frequencies would be overestimated when the temperature dependent material properties are not considered. The temperature change play an important role in the vibro-acoustic response of FGM plate, and the the corresponding peaks of the sound power level shift towards to lower frequency domain when the temperature rise increase.

\section{Acknowledgement}

The research was sponsored by the National Natural Science Foundation of China (no. 51575201).

\section{References}

[1] D.K. Jha, T. Kant, R.K. Singh, A critical review of recent research on functionally graded plates, Composite Structures, 96 (2013) 833-849.

[2] N. Chandra, S. Raja, K.V.N. Gopal, Vibro-acoustic response and sound transmission loss analysis of functionally graded plates, Journal of Sound and Vibration, 333 (2014) 5786-5802.

[3] T. Yang, Q. Huang, S. Li, Three-Dimensional Elasticity Solutions for Sound Radiation of Functionally Graded Materials Plates considering State Space Method, Shock Vib., 2016 (2015).

[4] B. Boley, A. Barber, Dynamic response of beams and plates to rapid heating, Journal of Applied Mechanics, 24 (1957) 413-425.

[5] P. Jeyaraj, N. Ganesan, C. Padmanabhan, Vibration and acoustic response of a composite plate with inherent material damping in a thermal environment, Journal of Sound and Vibration, 320 (2009) 322-338.

[6] Q. Geng, Y.M. Li, Analysis of Dynamic and Acoustic Radiation Characters for a Flat Plate under Thermal Environments, Int. J. Appl. Mech., 4 (2012) 1250028.

[7] X. Li, K. Yu, Vibration and acoustic responses of composite and sandwich panels under thermal environment, Composite Structures, 131 (2015) 1040-1049.

[8] M. Du, Q. Geng, Y.-m. Li, Vibrational and acoustic responses of a laminated plate with temperature gradient along the thickness, Composite Structures, (2016).

[9] H.-S. Shen, Z.-X. Wang, Assessment of Voigt and Mori-Tanaka models for vibration analysis of functionally graded plates, Composite Structures, 94 (2012) 2197-2208.

[10] J.N. Reddy, Mechanics of laminated composite plates and shells: theory and analysis, CRC press, 2004.

[11] F.J. Fahy, P. Gardonio, Sound and structural vibration: radiation, transmission and response, Academic press, 2007. 